\title{
Dynamic Responses of Reserve Carbohydrate Metabolism Under Carbon and Nitrogen Limitations in Saccharomyces cerevisiae
}

\author{
JEAN-LUC PARROU ${ }^{1}$, BRICE ENJALBERT ${ }^{1}$, LUCILE PLOURDE $^{1}$, ANNE BAUCHE $^{1}$, \\ BENJAMIN GONZALEZ ${ }^{1}$ AND JEAN FRANÇOIS ${ }^{1 *}$ \\ ${ }^{1}$ Centre de Bioingenierie Gilbert Durand, UMR-CNRS 5504, LA. INRA, INSA, Complexe Scientifique de Rangueil, \\ 31077 Toulouse Cedex 04, France
}

\begin{abstract}
The dynamic responses of reserve carbohydrates with respect to shortage of either carbon or nitrogen source was studied to obtain a sound basis for further investigations devoted to the characterization of mechanisms by which the yeast Saccharomyces cerevisiae can cope with nutrient limitation during growth. This study was carried out in well-controlled bioreactors which allow accurate monitoring of growth and frequent sampling without disturbing the culture. Under glucose limitation, genes involved in glycogen and trehalose biosynthesis ( $G L G 1, G S Y 1, G S Y 2$, $G A C 1, G L C 3, T P S 1)$, in their degradation $(G P H 1, N T H 1)$, and the typical stress-responsive CTT1 gene were coordinately induced in parallel with glycogen, when the growth has left the pure exponential phase and while glucose was still plentiful in the medium. Trehalose accumulation was delayed until the diauxic shift, although TPS1 was induced much earlier, due to hydrolysis of trehalose by high trehalase activity. In contrast, under nitrogen limitation, both glycogen and trehalose began to accumulate at the precise time when the nitrogen source was exhausted from the medium, coincidentally with the transcriptional activation of genes involved in their metabolism. While this response to nitrogen starvation was likely mediated by the stress-responsive elements (STREs) in the promoter of these genes, we found that these elements were not responsible for the co-induction of genes involved in reserve carbohydrate metabolism during glucose limitation, since $G L G 1$, which does not contain any STRE, was coordinately induced with GSY2 and TPS1. Copyright (C) 1999 John Wiley \& Sons, Ltd.
\end{abstract}

KEY WORDS - growth phases; nutrient limitation; reserve carbohydrates; stress; gene expression; Saccharomyces cerevisiae

\section{INTRODUCTION}

In the yeast Saccharomyces cerevisiae, cell division is tightly controlled at the level of the $\mathrm{G}_{1}$ phase of the cell cycle by environmental conditions including nutrient availability (Werner Washburne et al., 1993, 1996; Thomas and Hall, 1997). Before engaging in a subsequent round of division, cells must sense and integrate both intracellular and extracellular signals. The most usual experimental approach to investigate nutrient signalling pathways in growth control is the sudden removal of a nutrient essential to exponentially growing cells. As a consequence, haploid cells exit the mitotic

*Correspondence to: J. François, Centre de Bioingenierie Gilbert Durand, UMR-CNRS 5504, LA. INRA, INSA, Complexe Scientifique de Rangueil, 31077 Toulouse Cedex 04, France. Tel: 33-561559492; fax: 33-561559400; email: fran_jm@insa-tlse.fr cycle, enter the quiescent $\mathrm{G}_{0}$ state and develop stationary-phase characteristics which include accumulation of reserve carbohydrates and acquisition of thermotolerance (for reviews, see Werner Washburne et al., 1993, 1996). Diploid cells starved for nitrogen on a non-fermentable carbon source undergo meiosis and sporulation, whereas they sometimes develop pseudohyphal growth on a glucose-based medium (Gimeno et al., 1992; Kron, 1997).

The way in which yeast cells respond to the sudden removal of nutrient from the growth medium (i.e. nutrient starvation) is probably different from the way that they assess the progressive depletion of an essential nutrient during batch cultures (i.e. nutrient limitation), even though both conditions eventually lead to growth arrest. Indeed, Lillie and Pringle (1980) observed that as 
cells were depleting their supply of exogenous glucose, they started to accumulate glycogen when approximately half of the original glucose had been consumed. Other modifications probably occurred at the same time, including a reduction of the glycolytic flux and of the overall level of protein synthesis (François et al., 1987; Boucherie, 1985) and induction of heat shock and stress proteins (Werner Washburne et al., 1989; Bissinger et al., 1989; Boorstein and Craig, 1990; Varela et al., 1995). Using the DNA microarrays technique, DeRisi et al. (1997) illustrated the wide changes in expression patterns of known and uncharacterized genes during diauxic growth on a glucose-based rich medium. These physiological adaptations suggest that sophisticated dynamic sensing processes must exist to inform the cell of shortage of glucose and perhaps of other essential nutrients.

A critical step towards the characterization of these sensing processes was to define precisely the timing of the physiological responses with respect to the dynamic consumption of the limiting nutrient during growth. Such a study can be accurately performed in well-equipped fermenters which allow monitoring of growth parameters (temperature, $\mathrm{pH}$, gas-transfer, etc.) and high-frequency sampling without disturbing the culture, procedure which should avoid any misinterpretations from a two-sample analysis, i.e. one point in the exponential phase and the second one in the 'stationary' phase. Taking into account that glycogen and trehalose are hallmarks of changing nutritional conditions (Lillie and Pringle, 1980; ThompsonJaeger et al., 1991; François et al., 1991; Hardy et al., 1994; Winderickx et al., 1996), we reinvestigated the dynamic changes of these two aspects of metabolism during growth of yeast under carbonor nitrogen-limiting conditions. Since it was recently claimed that genes possessing one or several stress-responsive elements (STREs, Kobayashi and McEntee, 1993) share the same expression profile during growth on glucose (De Risi et al., 1997), the wide panel of genes investigated in this study allowed us to reconsider the requirement of STREs in gene induction.

\section{MATERIALS AND METHODS}

\section{Construction of yeast strains}

The Saccharomyces cerevisiae strains used in this work are listed in Table 1. The snfl $1:: H I S 3$ allele was constructed from $\mathrm{pBSK}-\mathrm{SNF} 1$ which contains a $3.3 \mathrm{~kb} E c o \mathrm{RI} / B a m \mathrm{HI}$ genomic fragment from SNF1 locus. Partial Bsml deletion resulted in the loss of the $3^{\prime}$ promoter region and most of the SNF1 catalytic domain (pJL23). Subsequent insertion of a HIS3 $1 \cdot 1 \mathrm{~kb}$ BamHI fragment into the Bcll site of the SNF1 regulatory domain yielded the final construct, pJL24. Construction of the $h \times k 2 \Delta:: H I S 3$ allele as carried out from pMR124, which contains a $2.43 \mathrm{~kb}$ $K p n \mathrm{I} / E c o$ RI genomic fragment with the $5^{\prime}$ part of the $H X K 2$ gene (gift from M. Rose and $\mathrm{K}$. D. Entian, University of Frankfurt, Germany). The KpnI/EcoRI fragment was subcloned into $K p n \mathrm{I} / E c o \mathrm{RI}$ digested pUC18 for convenient use in the following cloning steps (pJL9). As for $S N F 1$, the HIS3 $1.1 \mathrm{~kb} B a m H I$ fragment was introduced into the unique $\mathrm{Bcll}$ site of $\mathrm{HXK2}$ (pJL10) and further deletion of the $120 \mathrm{bp}$ EcoRV fragment from pJL10 yielded the final construct (pJL15). Gene disruption was carried out in the diploid strain JF326 according to the one-step replacement procedure (Rothstein, 1983), using the $3.5 \mathrm{~kb}$ EcoRI/BamHI fragment from pJL24 (SNF1 disruption) and the $3.4 \mathrm{~kb}$ EcoRI/BamHI fragment from pJL15 (HXK2 disruption). Correct disruption of $S N F 1$ and $H X K 2$ genes in $H_{I}{ }^{+}$colonies was confirmed by Southern blot analysis. Sporulation and dissection of diploids yielded the haploid strains JLP74-10A (snf14::HIS3 mutant) and JLP11-1A

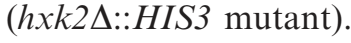

Construction and integration of plasmids bearing traductional fusions between GSY2, GACl, GPH1 or TPSI and the E. coli lacZ gene, and the pNL1 episomic plasmid carrying the NTH1-lacZ traductional fusion gene, have been described previously (Parrou et al., 1997). The

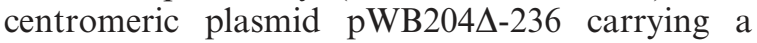
SSA3-lacZ fusion gene has been described by Boorstein and Craig (1990). Reinsertion of wild type LEU2 and HIS3 genes in JLP5-1A strain yielded the prototroph JLP48-3B strain. Crosses between haploid strains bearing lac $Z$ fusion genes and strains with $S N F 1, H X K 2$ or NTH1 null mutation were performed to obtain heterozygous diploid strains. These latter were sporulated and dissected, and haploid null mutant strains carrying a fusion gene were selected. Targeted integration of Ncol linearized pJL5 (GSY2-lacZ fusion gene) at the ura3 locus of RS13-58A-1 (tpk $k^{w} b c y 1$ mutant) yielded the strain JF908. 
Table 1. Yeast strains used in this work.

\begin{tabular}{|c|c|c|}
\hline Yeast strain & Genotype & Source \\
\hline KT1112 & a leu2 ura3 his3 & K. Tatchell \\
\hline JF326 & diploid from KT1112 & This study \\
\hline $\mathrm{X} 2180-1 \mathrm{~A}$ & a prototroph & YGSC $^{1}$ \\
\hline CENPK133-7D & a prototroph & P. Kötter \\
\hline JLP74-10A & a leu2 ura3 his3 snfts::HIS3 & This study \\
\hline JLP11-1A & 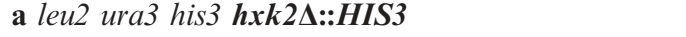 & This study \\
\hline YSN1 & a leu2 ura3 his3 can1 $\mathrm{gal}^{-}$nth1 $\Delta::$ LEU2 & H. Holzer ${ }^{2}$ \\
\hline RS13-58A-1 & $\begin{array}{l}\text { a leu2 ura3 his3 trp1 ade8 } \\
\text { tpk1 }^{\boldsymbol{w}} \text { tpk2::HIS3 tpk3::TRP1 bcy1::LEU2 }\end{array}$ & M. Wigler ${ }^{3}$ \\
\hline $11 \mathrm{a}-1 \mathrm{~A}$ & a leu2 his3 trp1 ade2 ade8 URA3::CTT1-lacZ & K. Tatchell \\
\hline JLP1-1D & a leu2 his3 trp1 ade2 can1 URA3::GSY2-lacZ & This study \\
\hline JF908 & 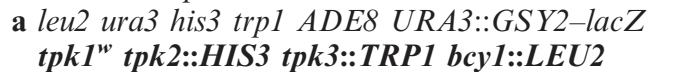 & This study \\
\hline JLP5-1A & a leu2 his3 URA3::GSY2-lacZ & This study \\
\hline JLP44-8A & a leu2 his3 URA3::GPH1-lacZ & This study \\
\hline JLP51-3D & a leu2 his3 URA3::GAC1-lacZ & This study \\
\hline JLP48-3B & a LEU2 HIS3 URA3::GSY2-lacZ & This study \\
\hline JLP75-4A & a leu2 his3 URA3::GSY2-lacZ snf1 1 ::HIS3 & This study \\
\hline JLP81-1C & 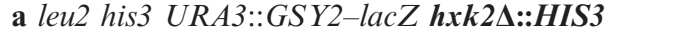 & This study \\
\hline JLP87-8A & a leu2 his3 URA3::TPS1-lacZ & This study \\
\hline JLP88-21A & a leu2 his3 GAL URA3::TPS1-lacZ NTH1 & This study \\
\hline JLP88-21B & a leu2 his3 GAL URA3::TPS1-lacZ nth1 & This study \\
\hline
\end{tabular}

${ }^{1}$ Mortimer and Johnston (1986). Genetics 113, 35-43; ${ }^{2}$ Nwaka et al. (1994). FEBS Lett. 344, 225-228;

${ }^{3}$ Cameron et al. (1988). Cell 53, 555-566.

\section{Reagents, media and culture conditions}

Yeast nitrogen base without amino acids and ammonium sulphate, Bacto peptone and yeast extract were from Difco laboratories. Chemicals for media preparation were from Merck or Prolabo and were of the purest grade available. Rich medium (YEP) contained (per litre) $10 \mathrm{~g}$ yeast extract, $20 \mathrm{~g}$ Bacto peptone and carbon sources at the indicated concentrations. Mineral medium (MIN) was prepared according to Johnston et al. (1977) and, unless otherwise stated, it contained $10 \mathrm{~g} / 1$ ammonium sulphate and $20 \mathrm{~g} / 1$ glucose. Mineral medium (YNB) contained $1.7 \mathrm{~g} / 1$ yeast nitrogen base without amino acids and ammonium sulphate, $5 \mathrm{~g}\left(\mathrm{NH}_{4}\right)_{2} \mathrm{SO}_{4}$ and $10 \mathrm{~g}$ glucose. It was supplemented with $100 \mu \mathrm{g} / 1$ of auxotrophic requirements. Both MIN and YNB were buffered at an initial $\mathrm{pH}$ of 5.8 by addition of $10 \mathrm{~g}$ succinic acid and $6 \mathrm{~g} \mathrm{NaOH} /$ litre. The 'cell factory medium' (CFM) was prepared according to Verduyn et al. (1992). In order to avoid any cross-reaction during autoclaving, stock solutions of YNB $(20 \times)$, vitamins and oligoelements $(1000 \times)$ were sterilized by filtration (Millipore nitrocellulose filters, $0.2 \mu \mathrm{m}$ pore size, $45 \mathrm{~mm}$ diameter). Stock solution of glucose $(500 \mathrm{~g} / 1)$ was sterilized by autoclaving for $30 \mathrm{~min}$ at $110^{\circ} \mathrm{C}$. Yeast precultures were carried out in half-litre flasks containing $100 \mathrm{ml}$ of the medium used in the subsequent batch fermenter. Cultures were performed at $30^{\circ} \mathrm{C}$ in 21 fermenters (Inceltech-SGI, France) with a 1.71 working volume, a $500 \mathrm{rpm}$ impeller speed and a $0.5 \mathrm{l} / \mathrm{min}$ airflow in order to maintain dissolved oxygen near saturation. For nitrogen starvation experiments, yeast cells grown in either YEP or YNB (20 g/l glucose and $5 \mathrm{~g} / 1$ ammonium sulphate) were rapidly collected by filtration at $\mathrm{OD}_{600} 0.5$ (Millipore nitrocellulose filters, $0.45 \mu \mathrm{m}$ pore size, $45 \mathrm{~mm}$ diameter), washed once with $10 \mathrm{ml}$ of YNB without nitrogen source and resuspended in the same volume of this nitrogen-free medium. Growth was monitored by measuring the absorbance of the culture at $600 \mathrm{~nm}$ with an Easyspec IV spectrophotometer (Safas SA, France). Growth curves were fitted using an exponential regression analysis (Excel 5.0 software), taking into account for calculation experimental points within the time window indicated 
by the arrows on the axis, i.e. during the apparent exponential phase. The prerequisite for this time window was to reach a correlation coefficient $\left(r^{2}\right)$ higher than 0.998 . The $\mu$ constant from the equation $\mathrm{OD}=\mathrm{b} \exp (\mu . \mathrm{t})$ of the fitting curve corresponds to the specific growth rate $\left(\mu=\ln 2 / T_{G}\right)$ where $T_{G}=$ the doubling time.

\section{Northern and Western blot analysis}

Western blot analysis of Tps1p was performed as described by Parrou et al. (1997). Signal quantification of autoradiographic film from Western analysis was performed using the Tina 2.0 PC Bas software. Total yeast RNAs were isolated following the procedure described in Siderius et al. (1997). Probes (656 bp EcoRI/ BamHI fragment from GSY2, 825 bp XmnI/NcoI fragment from $G L G 1,1245 \mathrm{bp} \mathrm{DraIII/NdeI} \mathrm{frag-}$ ment from NTH1, 1985 bp SacI/SacI fragment from LacZ and 1562 bp BamHI/HindIII fragment from $A C T 1)$ were $\alpha^{32} \mathrm{P}$-labelled using the random-priming method (Amersham Megaprime Kit).

\section{Enzymatic assays and analytical procedures}

For enzymatic assays, $10^{9}$ cell samples were quickly collected by filtration on nitrocellulose filters and frozen into liquid nitrogen. Preparation of crude extracts was performed as described by Parrou et al. (1997). Tre6P synthase activity was measured by the method of UDP formation (Vandercammen et al., 1989), except that the reaction was carried out at $42^{\circ} \mathrm{C}$ to reduce glycogen synthase interference. Glycogen synthase activity was measured in the presence of $0.25 \mathrm{~mm}$ UDP $\left[\mathrm{U}_{-}{ }^{14} \mathrm{C}\right]$ glucose without (active form) or with $10 \mathrm{~mm}$ Glc6P (total form) (François et al., 1988), and neutral trehalase activity was assayed as in Neves and François (1992). The total amount of proteins in the extracts was determined by the method of Bradford (1976) using bovine serum albumin as a standard. $2 \cdot 10^{8}$ cell samples were collected by centrifugation for the measurement of $\beta$-galactosidase activity (Rose and Botstein, 1983) and the quantitative determination of glycogen and trehalose (Parrou and François, 1997). Glucose and ammonium from the culture medium were measured using the Glucose Oxydase Reaction Kit (Sigma) and the Ammonium Electrode Analyser (Orion), respectively.

\section{RESULTS}

\section{Establishment of growth conditions and terminology}

In order to investigate the effects of specific nutrient limitations on the dynamics of glycogen and trehalose metabolism, an essential prerequisite was to define culture media limited respectively by carbon, nitrogen, sulphur or phosphorus source. With respect to terminology, we considered one of these four essential elements as 'limiting' for growth under discontinuous batch cultures, when it became exhausted from the medium while the others remained in large excess. The exhaustion of the limiting nutrient led to either a transient growth arrest (e.g. diauxic shift under glucose limitation, see Figure 1) or a sudden and continuous decay of the specific growth rate giving rise to a linear growth curve, followed by the entrance of cells into stationary phase (e.g. ammonium [see Figure 6], sulphur or phosphorus limitation). At this stage of growth, the cell biomass had to be roughly proportional to the initial concentration of the limiting nutrient.

We therefore found that the initial glucose concentration had to be lower than or equal to $10 \mathrm{~g} / 1$ in minimal media to ensure that it was limiting for growth of auxotrophic KT1112 and derivative strains. In contrast, the biomass of prototroph JLP48-3B, X2180-1A and CENPK133-7D strains increased almost proportionally with rising sugar concentrations, at least up to $40 \mathrm{~g} / \mathrm{l}$. From this observation, it was concluded that growth defects of auxotroph strains could result from impaired metabolic activities linked to their auxotrophies. As regards nitrogen source, nitrogen limitation on glucose-based MIN medium was obtained with an initial ammonium sulphate concentration lower than or equal to $2 \mathrm{~g} / \mathrm{l}$. In contrast, it was difficult to get proportional limitations for either phosphorus or sulphur sources, probably because of high intracellular stores of these compounds, which rendered yeast cells poorly sensitive to $\mathrm{P}_{\mathrm{i}^{-}}$or S-limitation. Hence, we did not investigate these nutritional conditions in this present work.

\section{Coordinated induction of genes but differential accumulation of glycogen and trehalose under glucose limitation}

Lillie and Pringle (1980) originally observed that yeast cells undergoing diauxic growth on glucose limited medium began to accumulate glycogen 

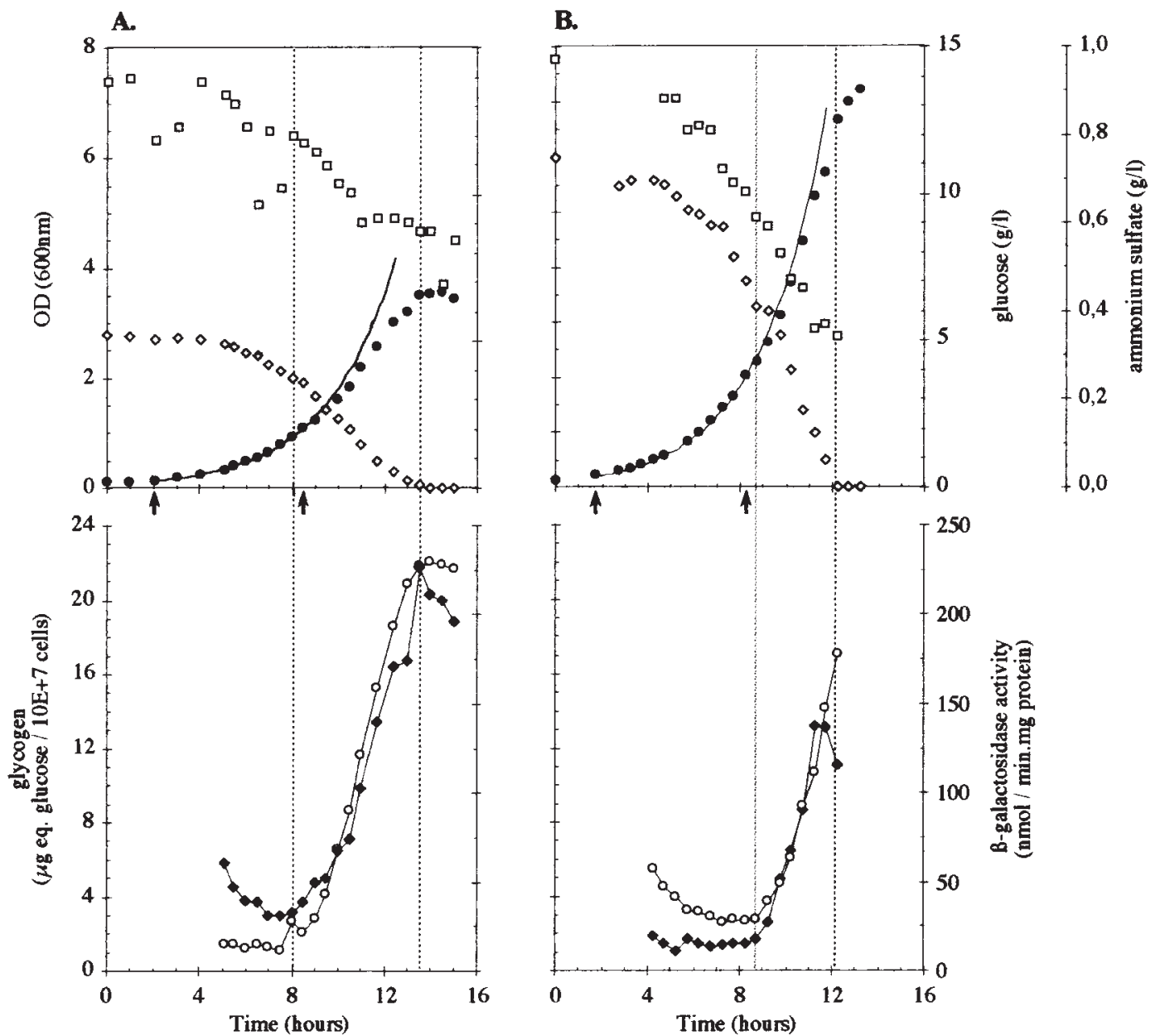

Figure 1. Glycogen levels and $\beta$-galactosidase specific activity from the GSY2-lac Z fusion gene during diauxic growth of the JLP48-3B strain on MIN with $5 \mathrm{~g}(\mathrm{~A})$ or $10 \mathrm{~g}(\mathrm{~B})$ glucose per litre. The culture medium was inoculated at initial $\mathrm{OD}_{600}=0.05$ (about $10^{6} \mathrm{cells} / \mathrm{ml}$ ) from an overnight shake flask preculture in the same medium. Cell density $(\bullet)$; glycogen $(\bigcirc)$; $\beta$-galactosidase activity $(\diamond)$; glucose $(\diamond)$; ammonium sulphate $(\square)$. Parameters from the equation of the fitting curve calculated from points between the arrows: $\mu=0.34, r^{2}=0.9983$ (A) and $\mu=0.35, r^{2}=0.9989$ (B).

when approximately $50 \%$ of glucose remained to be consumed. We confirmed their results and further showed that glycogen accumulation coincided with transcriptional induction of $G S Y 2$, as determined by $\beta$-galactosidase activity from the $G S Y 2$ lac $Z$ fusion gene, both reaching a maximum at the diauxic shift (Figure 1). This pattern was confirmed by Northern blot analysis of GSY2 transcripts, which peaked at the onset of glucose exhaustion from the medium (see Figure 3), suggesting a decline of transcription after this point. Identical expression patterns from lac $Z$ fusion genes (results not illustrated) were obtained in this study with genes encoding Glc3p (glycogen branching enzyme; Rowen et al., 1992; Thon et al., 1992), Gaclp (a regulatory protein of glycogen synthase; François et al., 1992), Gph1p (glycogen phosphorylase; Hwang et al., 1989) and with YPR184w encoding the glycogen debranching enzyme (unpublished results). It is also shown in Figure 3 that transcripts of GLG1 encoding glycogenin (Cheng et al., 1995) increased in parallel with GSY2 mRNAs.

With respect to genes involved in trehalose metabolism, we found that $\beta$-galactosidase activity from the TPS1-lacZ construct sharply rose in parallel with glycogen accumulation, despite a weak and steady increase during the exponential 
A.

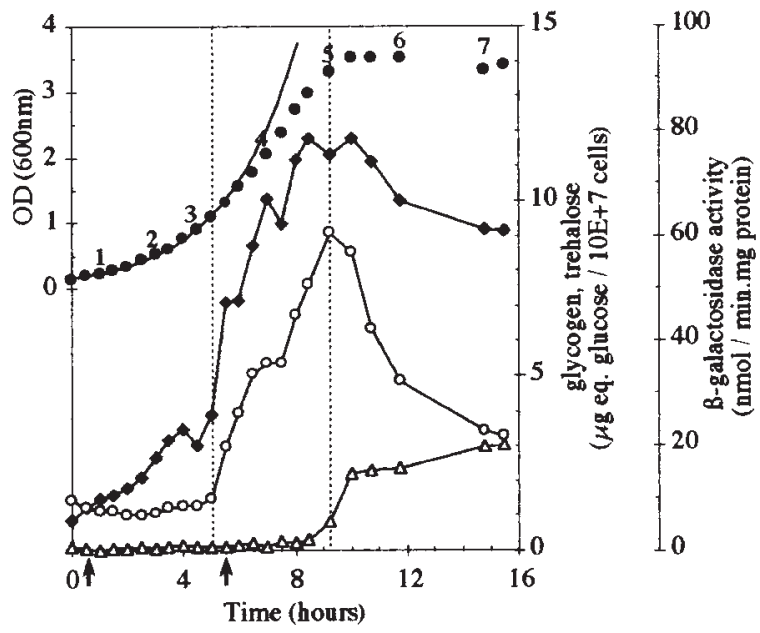

B.

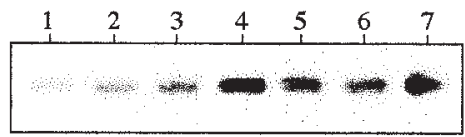

C.

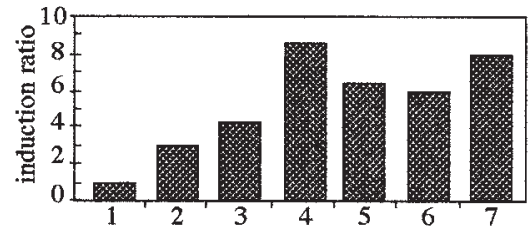

Figure 2. Glycogen and trehalose levels, $\beta$-galactosidase specific activity from the TPS1-lacZ fusion gene and Western blot analysis of Tpslp during diauxic growth of the JLP87-8A strain on YNB with $10 \mathrm{~g}$ glucose/litre. (A) Procedure and symbols as in Figure 1: trehalose $(\triangle)$. Parameters of the fitting curve: $\mu=0 \cdot 39 ; r^{2}=0 \cdot 9984$. (B) Samples (1-7) were taken during growth for immunoblot analysis with the primary antibody KH-1142 raised against the $56 \mathrm{kDa}$ subunit of Tre $6 P$ synthase (gift from J. Londesborough). (C) Induction factor relative to sample 1 after signal quantification from (B).

phase of growth (Figure 2A). The rise of TPS1 expression was quantitatively related to the accumulation of its product as revealed by Western blot analysis (Figures 2B, 2C). These results complemented previous data showing that Tre $6 P$ synthase/phosphatase complex activity significantly increased before the diauxic shift (François et al., 1991). Northern blot analysis additionally revealed an accumulation of NTH1 transcripts similar to that found for GSY2 mRNAs (Figure 3). This study was extended to genes that have been
A.

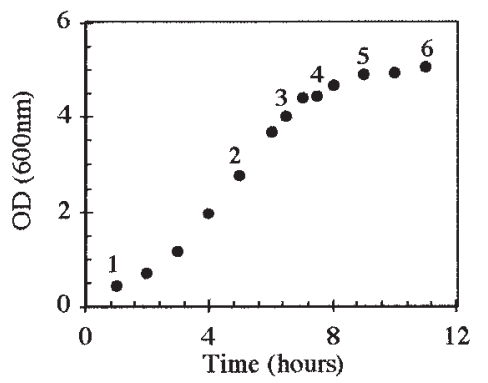

B.

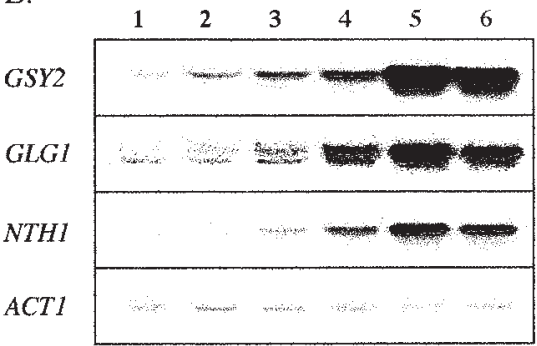

Figure 3. Northern blot analysis of GSY2, GLG1, NHT1 and $A C T 1 \mathrm{mRNAs}$ during diauxic growth of KT1112 on YEP with $10 \mathrm{~g}$ glucose/litre. (A) The culture was performed in a $500 \mathrm{ml}$ shake flask containing $100 \mathrm{ml}$ of medium. (B) $2 \cdot 10^{8}$ cell samples (1-6) were taken during growth for Northern blot analysis. The $A C T 1$ mRNA level was used as loading control.

considered to be induced between the diauxic shift and the entrance into stationary phase (Werner Washburne et al., 1996), namely the stressresponsive gene $C T T 1$ encoding the cytosolic catalase, and $S S A 3$ encoding an Hsp70p. We found that the expression profile from the CTT1-lacZ fusion gene perfectly matched that of TPS1, while $S S A 3-l a c Z$ expression clearly increased after the diauxic shift (results not shown), as was previously reported for the $S S A 3-l a c Z$ construct and $S S A 3$ transcripts (Boorstein and Craig, 1990; Fuge et al., 1994).

As shown in Figure 2, trehalose began to accumulate only at the onset of the diauxic shift after the induction of TPS1, which is involved in its biosynthesis. Since NTH1 was coordinately induced with TPS1, we investigated whether the trehalase was not responsible for the delay of trehalose accumulation using a nthl null mutant. This mutant strain exhibited wild-type TPS1 expression, but it accumulated trehalose in parallel with glycogen in contrast to wild-type strains (Figure 4). 


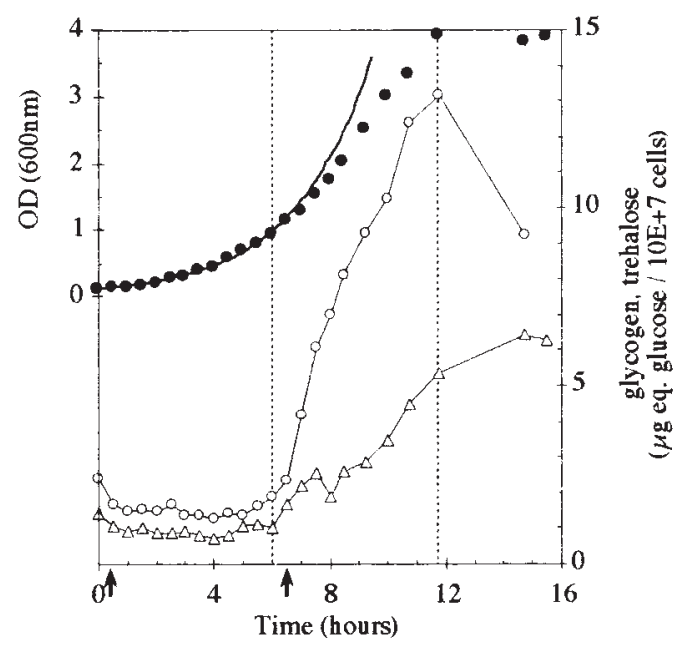

Figure 4. Glycogen and trehalose levels during diauxic growth of JLP88-21B (nth1 null mutant) on YNB with $10 \mathrm{~g}$ glucose/ litre. Procedure and symbols as in Figure 1; trehalose $(\triangle)$. Parameters of the fitting curve: $\mu=0 \cdot 38, r^{2}=0 \cdot 9989$.

\section{Glucose effect on gene expression}

It can be seen in Figure 1 that the higher the initial glucose concentration, the lower the level of GSY2 expression and the content of glycogen at the diauxic shift of growth in MIN. Note that such an inhibitory effect was previously reported for CTT1-lacZ expression on YEP with 20 and $100 \mathrm{~g}$ glucose/litre (Bissinger et al., 1989). As illustrated in Table 2, this inhibitory effect was also observed in other media (YNB and YEP) and in nullmutants for $S N F 1$ and $H X K 2$, two genes involved in glucose repression (for a review, see Ronne, 1995). It also occurred, although to a lower extent, in a strain with lowered cAMP-dependent protein kinase (tpk $1^{w}$ tpk2 tpk3 bcyl mutant). In contrast, the inhibitory effect was not observed in galactose-based medium, indicating that it was glucose-specific.

The medium $\mathrm{pH}$ is not the trigger of glycogen accumulation under glucose-limited conditions

Throughout these studies, we systematically found that glycogen accumulation occurred during a transition period between the exit from an exponential phase and the entrance into the diauxic shift (Figures 1, 2, 4). This period corresponded to a decay of specific rate of both growth and glucose consumption (not shown). Since the acidification of the culture medium is known to cause inhibition of cell proliferation (Jones et al., 1981), the possibility that $\mathrm{pH}$ could be a common denominator between glycogen accumulation and the growth inhibition observed during this transition period was examined. It is, however, unlikely that a drop in $\mathrm{pH}$ could be the trigger of this phenomenon, since a decrease of only 0.4 unit was recorded during the growth in a medium initially buffered at pH 5.8 (Figures 1, 2, 4, parameter not plotted).

Table 2. Glucose effect on $\beta$-galactosidase activity from GSY2-lacZ fusion gene.

\begin{tabular}{|c|c|c|c|c|c|c|c|c|c|}
\hline \multirow[b]{3}{*}{ Lane } & \multirow[b]{3}{*}{ Strain } & \multirow[b]{3}{*}{ Genotype } & \multirow[b]{3}{*}{ Medium } & \multicolumn{6}{|c|}{ Sugar concentration $(\mathrm{g} / \mathrm{l})$} \\
\hline & & & & 5 & 10 & 20 & 40 & 60 & 100 \\
\hline & & & & \multicolumn{6}{|c|}{$\beta$-Galactosidase activity (nmol/min.mg protein) } \\
\hline 1 & JLP48-3B & WT & MIN & 210 & 150 & 90 & - & - & - \\
\hline 2 & JLP5-1A & WT & YNB & 125 & 70 & 60 & 50 & - & - \\
\hline 3 & JLP5-1A & WT & YEP & 175 & 140 & 100 & 40 & - & - \\
\hline 4 & JLP5-1A & WT & YEP & - & 85 & 48 & 24 & - & 12 \\
\hline 5 & JLP81-1C & $h x k 2$ & YEP & - & 100 & 70 & 40 & - & 32 \\
\hline 6 & JF908 & $t p k^{w}, b c y l$ & YEP & - & 190 & 170 & 150 & - & 130 \\
\hline 7 & JLP5-1A & WT & YEP & - & - & 64 & 48 & 36 & - \\
\hline 8 & JLP5-1A & WT & YEPGal & - & - & 256 & 260 & 264 & - \\
\hline
\end{tabular}

Glucose was used in all experiments except in lane 8, where it was replaced by galactose. Because of variability of $\beta$-galactosidase activity between independent experiments, the wild-type strain JLP5-1A was systematically included as a control in the groups of experiments reported in lanes 4-6 and 7-8. For one specific condition, the value reported in the Table is the mean of five consecutive points performed in $1 \mathrm{~h}$ intervals during the diauxic shift, with a standard deviation less than $10 \%$. 

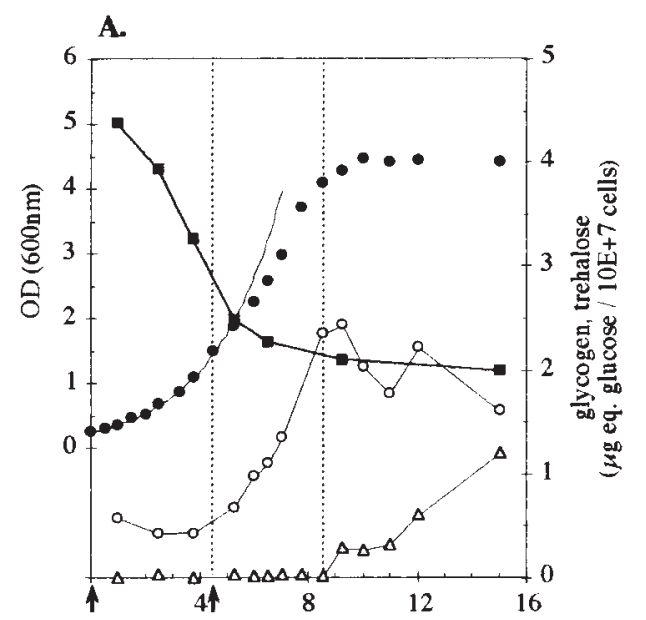

7
6
5
4
3
2
1
0

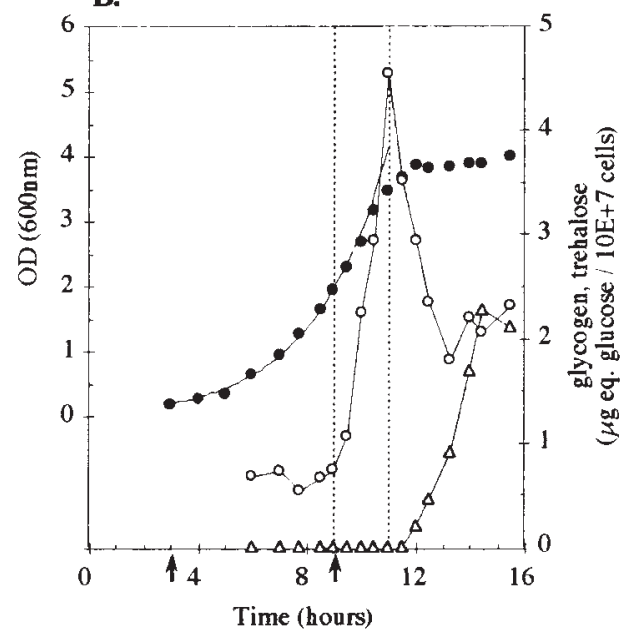

Figure 5. Effect of medium $\mathrm{pH}$ on glycogen induction during diauxic growth of X2180-1A strain on CFM with $10 \mathrm{~g}$ glucose/ litre. The $\mathrm{pH}$ of the medium was uncontrolled (A) or maintained constant at $5 \cdot 0$ (B). Procedure and symbols as in Figure 1; trehalose $(\triangle)$; $\mathrm{pH}(\boldsymbol{\square})$. Parameters of the fitting curve: $\mu=0.40, r^{2}=0.9984$ (A) and $\mu=0.37, r^{2}=0.999$ (B).

Nonetheless, experiments were performed using a mineral medium (CFM) in which the $\mathrm{pH}$ was either not controlled or kept at a constant value during growth. In a preliminary experiment, we confirmed that the kinetics of reserve carbohydrate accumulation in CFM initially buffered at $\mathrm{pH} 5.8$ was the same as in buffered MIN and YNB (not illustrated). Under uncontrolled $\mathrm{pH}$, glycogen accumulation began very early as cell proliferation ceased to be exponential, when the external $\mathrm{pH}$ decreased below $4 \cdot 0$ (Figure 5A). When the $\mathrm{pH}$ of the medium was kept at a constant value of $5 \cdot 0$, glycogen started to accumulation before the diauxic shift, while the growth was still exponential; in this case, the rupture in the growth pattern was clearly delayed (Figure 5B). This latter growth condition also resulted in a four-fold increase in the cells' capacity to accumulate glycogen, e.g. $2 \mu \mathrm{g}$ eq. glucose per $10^{7}$ cells per hour as compared to $0.5 \mu \mathrm{g}$ under uncontrolled $\mathrm{pH}$. These results suggest that extracellular $\mathrm{pH}$ negatively affects the rate of activities involved in glycogen metabolism. However, it is unlikely that the $\mathrm{pH}$ affects glycogen accumulation indirectly by influencing the growth rate of the population, since cells growing slowly on gluconeogenic substrates display a very strong accumulation of reserve carbohydrates (unpublished results).

\section{Reserve carbohydrates induced in response to nitrogen limitation}

In contrast to glucose limitation, exponentially growing cells on a nitrogen-limited medium coordinately induced GSY2 and glycogen accumulation at the precise moment when the exogenous nitrogen source was exhausted (Figure 6). Identical results were obtained with media limited by 1 or $2 \mathrm{~g}$ ammonium sulphate/litre (not shown). Interestingly, yeast cells accumulated high levels of glycogen, while the transcriptional activation of $G S Y 2$ was lower than under glucose limitation (cf. Figures 1 and 6). Another notable peculiarity under nitrogen limitation was the concomitant accumulation of trehalose and glycogen.

Our results suggest that the sudden lack of nitrogen which arose during growth could trigger these genetic and metabolic changes. Similar results could therefore be expected when exponential growing cells on glucose experience nitrogen starvation. Indeed, it can be seen in Figure 7 that glycogen and trehalose accumulation started almost immediately after the shift to nitrogen-free medium, with a kinetic profile quantitatively comparable to that found under nitrogen limitation (Figure 6). This was accompanied by an increase of $\beta$-galactosidase from the GSY2-lac Z fusion gene (Figure 7), by an accumulation of Gsy2p and its activation, as indicated respectively by a rise of the total form of glycogen synthase activity and an increase in the ratio between the active and total form (not shown). Similar increases for TPS1 expression from the TPS1-lacZ fusion gene and for Tre6 $P$ synthase activity (not shown) were 


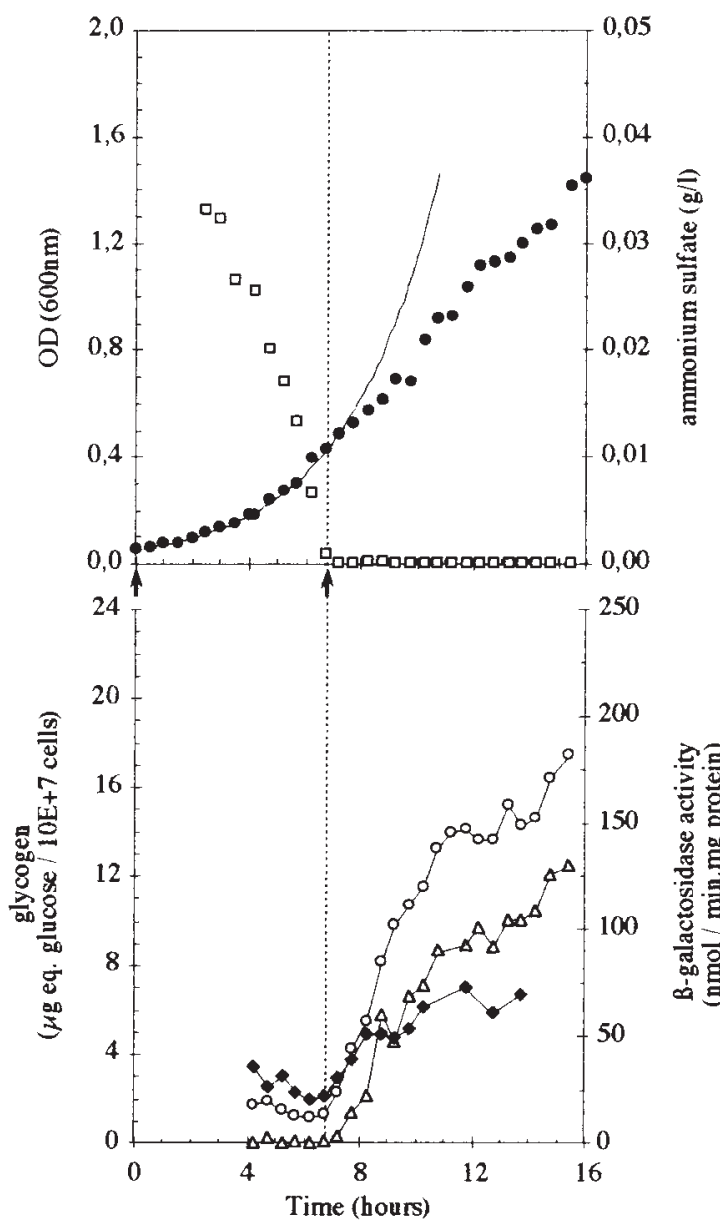

Figure 6. Glycogen and trehalose levels and $\beta$-galactosidase specific activity from the $G S Y 2-l a c Z$ fusion gene during growth of the JLP48-3B strain on MIN with $0.05 \mathrm{~g}$ ammonium sulphate/litre. Procedure and symbols as in Figure 1; trehalose $(\triangle)$. Parameters of the fitting curve: $\mu=0.31, r^{2}=0.9956$.

observed. In addition, a sudden 30-50\% drop of neutral trehalase activity was seen 10 min after the shift, followed by a recovery to its initial value after $2 \mathrm{~h}$ (Figure 7). This subsequent rise may be explained by the transcriptional activation of $N T H 1$, which occurred in parallel with GSY2 and TPS1 (not illustrated).

\section{DISCUSSION}

Using glycogen and trehalose as typical markers of changing environmental conditions, we report that reserve carbohydrate metabolism in Saccharomyces cerevisiae is differentially controlled by nitrogen and glucose limitations. While we did not find

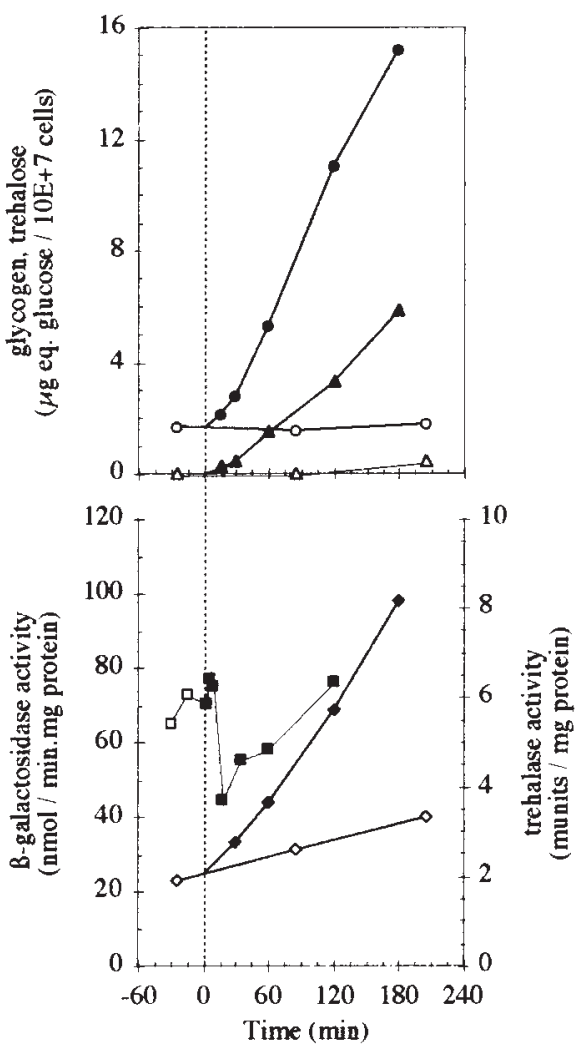

Figure 7. Effect of nitrogen starvation on glycogen and trehalose levels, and on specific activities of $\beta$-galactosidase (GSY2-lacZ fusion gene) and trehalase. Exponential growing cells of JLP48-3B strain were transferred at time zero from YNB to nitrogen-free medium, as described in Materials and Methods. Symbols: glycogen $(\bigcirc, \bullet)$; trehalose $(\triangle, \boldsymbol{\Delta})$; specific activities of $\beta$-galactosidase $(\diamond, \diamond)$ and trehalase $(\square, \mathbf{\square})$; control (empty symbols), nitrogen starvation (full symbols).

any obvious factor responsible for the activation of this metabolism under glucose limitation (see below), complete exhaustion of the nitrogen source under nitrogen limitation appeared to be the primary condition to trigger the activation of both glycogen and trehalose metabolism. As compared to glucose limitation, other differences were a lower transcriptional activation of genes, despite a large and concomitant accumulation of both reserve carbohydrates. This large accumulation could not be solely accounted for by the moderate induction of GSY2 and TPS1, especially as there was a simultaneous activation of GPHI and NTH1, which probably resulted in recycling of glycogen and trehalose, as previously reported under other stress conditions (Parrou et al., 1997). 
The net accumulation of reserve carbohydrates therefore implies additional mechanisms, including activation of glycogen synthase and inactivation of neutral trehalase, and increased availability of glucose- $6 P$, the substrate of Tre $6 P$ synthase and a positive effector of glycogen synthase (François et al., 1988; Huang et al., 1997).

An astonishing feature of cellular responses to glucose limitation reported some 20 years ago by Lillie and Pringle (1980), was that glycogen accumulation began when approximately half of the glucose remained to be consumed, regardless of its initial concentration in the medium. Interestingly, we observed in most of the experiments reported in this work that glycogen began to accumulate almost coincidentally with a premature exit from the exponential phase, suggesting a link between growth inhibition and the triggering of glycogen accumulation. However, in some experiments (e.g. controlled $\mathrm{pH}$ ) or during growth in galactoselimited medium (unpublished results), glycogen accumulation was initiated while the growth was still perfectly exponential. Therefore, these two events do not appear necessarily to be linked and the common idea that reduction of the specific growth rate causes accumulation of glycogen should not be considered as a general rule (Lillie and Pringle, 1980; François et al., 1987; Larsson et al., 1997). This work and previous reports (Lillie and Pringle, 1980; François et al., 1987, 1991; San Miguel and Argüelles, 1994) have shown that trehalose accumulation only started at the diauxic shift. Using a nthl null mutant, we could show that this delay is due to high trehalase activity which overcomes Tre $6 P$ synthase/phosphatase complex activity during the transition phase of growth. In addition, the lack of neutral trehalase also resulted in measurable amounts of trehalose during the exponential phase, revealing the basal activity of Tre $6 P$ synthase, which is probably necessary for the steady-state formation of trehalose- $6 P$, a key regulatory molecule controlling the glycolytic flux in yeast (Blazquez et al., 1993; Thevelein and Hohmann, 1995). Since we observed that the transcription of NTHI is enhanced during this transition and maximal at the entrance of the diauxic shift, the drop of neutral trehalase activity (François et al., 1987; San Miguel and Argüelles, 1994) suggests a strong inactivation of this enzyme, by a mechanism which remains to be clarified. A potential physiological significance of high levels of 'inactive' trehalase, as well as glycogen phosphorylase, during and after the diauxic shift of growth, is to allow yeast cells to rapidly resume growth under favourable conditions by mobilization of their endogenous stores.

The coordinated induction of genes involved in glycogen metabolism was previously inferred from the coordinated expression of one specific gene with glycogen accumulation (Hwang et al., 1989; François et al., 1992; Thon et al., 1992; Rowen et al., 1992; Hardy et al., 1994; Ni and LaPorte, 1995). The use of fermentation technology for accurate monitoring of growth and sampling allowed us to further reinforce this observation for the wide panel of genes involved in glycogen metabolism, i.e. GSY2, GPH1, GLC3, GAC1, GLG1 and YPR184w, encoding the debranching enzyme (M. A. Teste and J. François, unpublished results). A coordinated induction during the diauxic growth, never reported so far, was also observed for TPS1, NTH1 and CTT1, respectively involved in trehalose biosynthesis, trehalose degradation and the general stress response. Notable discrepancies between our results and literature data need to be pointed out. The first concerns the absence of GPH1 induction in the work of De Risi et al. (1997), while this gene has been shown in many reports to be induced before the diauxic shift (Hwang et al., 1989; Thon et al., 1992; François et al., 1992). The most striking difference was the decrease of TPS1 mRNAs from the exponential to the 'stationary' phase reported by Winderickx et al. (1996), which is in deep contradiction with a strong increase of $\beta$-galactosidase activity from the TPS1-lacZ fusion gene (Figure 2A; Winderickx et al., 1996), of TPS1 transcripts and of Tpslp between the exponential and the diauxic shift of growth (Figures 2B, 2C; François et al., 1987; Vuorio et al., 1993).

The term 'stationary phase' should be definitely avoided in designating this panel of genes (Werner Washburne et al., 1996), since they were clearly induced much earlier than the 'post-diauxic shift' SSA3 gene (Boorstein and Craig, 1990) and the truly 'stationary phase' SNZ1 gene (Braun et al., 1996). Moreover, the specific induction of this class of genes while glucose was still abundant in the medium, and their maximal expression at the onset of the diauxic shift, argued against the claim that these genes are under 'catabolic repression' (Vuorio et al., 1993; Kopp et al., 1993; Nwaka et al., 1995a, b; Hardy et al., 1994; Winderickx et al., 1996), since typical glucose-repressed genes are solely derepressed after the complete exhaustion of glucose from the medium (Ronne, 1995; 
De Risi et al., 1997). Even though catabolite repression is indeed inappropriate, we noticed a strong negative correlation between initial glucose concentration and reserve carbohydrate metabolism, but we do not yet have a clear explanation for this specific 'glucose effect'.

A common denominator for most of the genes studied in this work was the presence of at least one STRE in their promoter region. This cis-acting element was considered to be necessary to mediate transcriptional activation of genes in response to various stress conditions (for reviews, see Ruis and Schüller, 1995; Siderius and Mager, 1997), including nutrient starvation, temperature, oxidative, osmotic and ethanol shocks. In particular, this STRE-dependent activation was well illustrated for CTT1 and GSY2 in response to nitrogen starvation (Marchler et al., 1993; Ni and LaPorte, 1995; unpublished results). It was also proposed that GSY2 and HSP12 induction during diauxic growth on glucose was dependent on STREs present in their promoter (Ni and LaPorte, 1995; Varela et al., 1995). De Risi et al. (1997) extended this statement to 'STRE-containing' genes, since they showed their co-induction before the diauxic shift of growth. However, at least with respect to genes involved in reserve carbohydrate metabolism, we do not share this assertion because GLG1, which does not harbour any STRE, was induced in parallel with GSY2 and TPS1. Furthermore, preliminary data based on identical experimental procedures showed that GSY2 was still properly induced after removal of the two STREs by site-directed mutagenesis. The co-induction of this panel of genes is not triggered by inactivation of the cAPK pathway (Parrou et al., manuscript in preparation) and work is currently under way to identify the molecular mechanisms underlying this specific induction during the diauxic growth on glucose.

\section{ACKNOWLEDGEMENTS}

We thank J. Cannon (Missouri, USA), K. Tatchell (Shreveport, USA), P. K. Hwang (San Francisco, USA), E. Craig (Madison, USA) and J. Londesborough (Helsinki, Finland) for providing plasmids or antibodies. We are grateful to $\mathrm{C}$. Gancedo, M. A. Blazquez, G. Goma and V. Paquet for fruitful discussions during this work and to M. A. Teste for her excellent technical assistance. This work was supported in part by the 'Action Integrée France-Espagne' No. 94086 and in part by the Commission of the European Union (programme Cell Factories No. BIO4.CT95.132).

\section{REFERENCES}

Bissinger, P. H., Wieser, R., Hamilton, B. and Ruis, H. (1989). Control of Saccharomyces cerevisiae catalase $\mathrm{T}$ gene (CTT1) expression by nutrient supply via the RAS-cyclic AMP pathway. Mol. Cell. Biol. 9, 13091315.

Blazquez, M. A., Lagunas, R., Gancedo, C. and Gancedo, J. M. (1993). Trehalose-6-phosphate, a new regulator of yeast glycolysis that inhibits hexokinases. FEBS Lett. 329, 51-54.

Boorstein, W. R. and Craig, E. A. (1990). Transcriptional regulation of $S S A 3$, an HSP70 gene from Saccharomyces cerevisiae. Mol. Cell. Biol. 10, 3262 3267.

Boucherie, H. (1985). Protein synthesis during transition and stationary phases under glucose limitation in Saccharomyces cerevisiae. J. Bacteriol. 161, 385-392.

Bradford, M. M. (1976). A rapid and sensitive method for the quantitation of microgram quantities of protein utilizing the principle of protein-dye binding. Anal. Biochem. 72, 248-254.

Braun, E. L., Fuge, E. K., Padilla, P. A. and Werner, W. M. (1996). A stationary-phase gene in Saccharomyces cerevisiae is a member of a novel, highly conserved gene family. J. Bacteriol. 178, 6865-6872.

Cheng, C., Mu, J., Farkas, I., Huang, D., Goebl, M. G. and Roach, P. J. (1995). Requirement of the selfglucosylating initiator proteins Glg1p and Glg2p for glycogen accumulation in Saccharomyces cerevisiae. Mol. Cell. Biol. 15, 6632-6640.

De Risi, J. L., Iyer, V. R. and Brown, P. O. (1997). Exploring the metabolic and genetic control of gene expression on a genomic scale. Science 278, 680-686.

François, J., Eraso, P. and Gancedo, C. (1987). Changes in the concentration of cAMP, fructose 2,6bisphosphate and related metabolites and enzymes in Saccharomyces cerevisiae during growth on glucose. Eur. J. Biochem. 164, 369-373.

François, J., Villanueva, M. E. and Hers, H. G. (1988). The control of glycogen metabolism in yeast. 1. Interconversion in vivo of glycogen synthase and glycogen phosphorylase induced by glucose, a nitrogen source of uncouplers. Eur. J. Biochem. 174, 551-559.

François, J., Neves, M. J. and Hers, H. G. (1991). The control of trehalose biosynthesis in Saccharomyces cerevisiae: evidence for a catabolite inactivation and repression of trehalose-6-phosphate synthase and trehalose-6-phosphate phosphatase. Yeast 7, 575-587.

François, J. M., Thompson, J. S., Skroch, J., Zellenka, U., Spevak, W. and Tatchell, M. (1992). GACl may encode a regulatory subunit for protein phosphatase type 1 in Saccharomyces cerevisiae. EMBO J. 11, 87-96. 
Fuge, E. K., Braun, E. L. and Werner, W. M. (1994) Protein synthesis in long-term stationary-phase cultures of Saccharomyces cerevisiae. J. Bacteriol. 176, $5802-5813$.

Gimeno, C. J., Ljungdahl, P. O., Styles, C. A. and Fink, G. R. (1992). Unipolar cell divisions in the yeast $S$. cerevisiae lead to filamentous growth: regulation by starvation and RAS. Cell 68, 1077-1090.

Hardy, T. A., Huang, D. and Roach, P. J. (1994). Interactions between cAMP-dependent and SNF1 protein kinases in the control of glycogen accumulation in Saccharomyces cerevisiae. J. Biol. Chem. 269, 27907-27913.

Huang, D., Wilson, W. A. and Roach, P. J. (1997). Glucose-6-P control of glycogen synthase phosphorylation in yeast. J. Biol. Chem. 272, 22495-22501.

Hwang, P. K., Tugendreich, S. and Fletterick, R. J. (1989). Molecular analysis of GPH1, the gene encoding glycogen phosphorylase in Saccharomyces cerevisiae. Mol. Cell. Biol. 9, 1659-1666.

Johnston, G. C., Pringle, J. R. and Hartwell, L. H. (1977). Coordination of growth with cell division in the yeast Saccharomyces cerevisiae. Exp. Cell. Res. 105, 79-98.

Jones, R. P., Pamment, N. and Greenfield, P. F. (1981) Alcohol fermentation by yeasts - the effect of environmental and other variables. Process. Biochem. April/ May, 42-49.

Kobayashi, N. and McEntee, K. (1993). Identification of cis and trans components of a novel heat shock stress regulatory pathway in Saccharomyces cerevisiae. Mol. Cell. Biol. 13, 248-256.

Kopp, M., Muller, H. and Holzer, H. (1993). Molecular analysis of the neutral trehalase gene from Saccharomyces cerevisiae. J. Biol. Chem. 268, 4766-4774.

Kron, S. J. (1997). Filamentous growth in budding yeast. Trends Microbiol. 5, 450-454.

Larsson, C., Nilsson, A., Blomberg, A. and Gustafsson, L. (1997). Glycolytic flux is conditionally correlated with ATP concentration in Saccharomyces cerevisiae: a chemostat study under carbon- or nitrogen-limiting conditions. J. Bacteriol. 179, 7243-7250.

Lillie, S. H. and Pringle, J. R. (1980). Reserve carbohydrate metabolism in Saccharomyces cerevisiae: responses to nutrient limitation. J. Bacteriol. 143, 1384-1394.

Marchler, G., Schüller, C., Adam, G. and Ruis, H. (1993). A Saccharomyces cerevisiae UAS element controlled by protein kinase A activates transcription in response to a variety of stress conditions. EMBO J. 12, 1997-2003.

Neves, M. J. and François, J. (1992). On the mechanism by which a heat shock induces trehalose accumulation in Saccharomyces cerevisiae. Biochem. J. 288, 859864.

Ni, H. T. and LaPorte, D. C. (1995). Response of a yeast glycogen synthase gene to stress. Mol. Microbiol. 16, $1197-1205$.
Nwaka, S., Kopp, M. and Holzer, H. (1995a). Expression and function of the trehalase genes NTH1 and YBR0106 in Saccharomyces cerevisiae. J. Biol. Chem. 270, 10193-10198.

Nwaka, S., Mechler, B., Destruelle, M. and Holzer, H. (1995b). Phenotypic features of trehalase mutants in Saccharomyces cerevisiae. FEBS Lett. 360, 286-290.

Parrou, J. L. and François, J. (1997). A simplified procedure for a rapid and reliable assay of both glycogen and trehalose in whole yeast cells. Anal. Biochem. 248, 186-188.

Parrou, J. L., Teste, M. A. and François, J. (1997). Effects of various types of stress on the metabolism of reserve carbohydrates in Saccharomyces cerevisiae: genetic evidence for a stress-induced recycling of glycogen and trehalose. Microbiology 143, 1891-1900.

Ronne, H. (1995). Glucose repression in fungi. Trends Genet. 11, 12-17.

Rose, M. and Botstein, D. (1983). Construction and use of gene fusions to lac $Z$ ( $\beta$-galactosidase) that are expressed in yeast. Methods Enzymol. 101, 167-180.

Rothstein, R. J. (1983). One-step disruption in yeast. Methods Enzymol. 101, 202-211.

Rowen, D. W., Meinke, M. and LaPorte, D. C. (1992). GLC3 and GHAl of Saccharomyces cerevisiae are allelic and encode the glycogen branching enzyme. Mol. Cell. Biol. 12, 22-29.

Ruis, H. and Schüller, C. (1995). Stress signaling in yeast. Bioessays 17, 959-965.

San Miguel, P. and Argüelles, J. C. (1994). Differential changes in the activity of cytosolic and vacuolar trehalases along the growth cycle of Saccharomyces cerevisiae. Biochim. Biophys. Acta 1200, 155-160.

Siderius, M. and Mager, W. H. (1997). General stress response: in search of a common denominator. In Hohmann, S. and Mager, W. H. (Eds), Yeast Stress Responses, R. G. Landes Company, pp. 213-230.

Siderius, M., Rots, E. and Mager, W. H. (1997). Highosmolarity signalling in Saccharomyces cerevisiae is modulated in a carbon-source-dependent fashion. Microbiology 143, 3241-3250.

Thevelein, J. M. and Hohmann, S. (1995). Trehalose synthase: guard to the gate of glycolysis in yeast? Trends Biochem. Sci. 20, 3-10.

Thomas, G. and Hall, M. N. (1997). TOR signalling and control of cell growth. Curr. Opin. Cell Biol. 9, $782-787$.

Thompson Jaeger, S., Francois, J., Gaughran, J. P. and Tatchell, K. (1991). Deletion of SNF1 affects the nutrient response of yeast and resembles mutations which activate the adenylate cyclase pathway. Genetics 129, 697-706.

Thon, V. J., Vigneron, L. C., Marianne, P. T. Montreuil, J., Decq, A., Rachez, C., Ball, S. G. and Cannon, J. F. (1992). Coordinate regulation of glycogen metabolism in the yeast Saccharomyces cerevisiae. Induction of glycogen branching enzyme. J. Biol. Chem. 267, 15224-15228. 
Vandercammen, A., François, J. and Hers, H. G. (1989). Characterization of trehalose-6-phosphate synthase and trehalose-6-phosphate phosphatase of Saccharomyces cerevisiae. Eur. J. Biochem. 182, 613-620.

Varela, J. C., Praekelt, U. M., Meacock, P. A., Planta, R. J. and Mager, W. H. (1995). The Saccharomyces cerevisiae HSP12 gene is activated by the highosmolarity glycerol pathway and negatively regulated by protein kinase A. Mol. Cell. Biol. 15, 6232-6245.

Verduyn, C., Postma, E., Scheffers, W. A. and Van, D. J. (1992). Effect of benzoic acid on metabolic fluxes in yeast: a continuous-culture study on the regulation of respiration and alcoholic fermentation. Yeast $\mathbf{8}$, $501-517$

Vuorio, O. E., Kalkkinen, N. and Londesborough, J. (1993). Cloning of two related genes encoding the $56 \mathrm{kDa}$ and $123 \mathrm{kDa}$ subunits of trehalose synthase from the yeast Saccharomyces cerevisiae. Eur. J. Biochem. 216, 849-861.
Werner Washburne, M., Becker, J., Kosic, S. J. and Craig, E. A. (1989). Yeast Hsp70 RNA levels vary in response to the physiological status of the cell. J. Bacteriol. 171, 2680-2688.

Werner Washburne, M., Braun, E., Johnston, G. C. and Singer, R. A. (1993). Stationary phase in the yeast Saccharomyces cerevisiae. Microbiol. Rev. 57, 383401.

Werner Washburne, M., Braun, E. L., Crawford, M. E. and Peck, V. M. (1996). Stationary phase in Saccharomyces cerevisiae. Mol. Microbiol. 19, 1159-1166.

de Winderickx, J. W. J., Crauwels, M., Hino, A., Hohmann, S., Van, D. P. and Thevelein, J. M. (1996). Regulation of genes encoding subunits of the trehalose synthase complex in Saccharomyces cerevisiae: novel variations of STRE-mediated transcription control? Mol. Gen. Genet. 252, 470-482. 